\title{
DIVISION XII / COMMISSION 46 / PROGRAM GROUP INTERNATIONAL SCHOOLS FOR YOUNG ASTRONOMERS
}

\author{
CHAIR \\ VICE-CHAIR \\ PAST CHAIR
}

\author{
Jean-Pierre De Greve \\ Kam-Ching Leung \\ Michèle Gerbaldi
}

\section{TRIENNIAL REPORT 2006 - 2009}

\section{Introduction}

The IAU Commission 46 Program Group International Schools for Young Astronomers (ISYA) was created in 1967 (Gerbaldi 2008). During the period 2006 till August 2008 two ISYAs took place, one in Malaysia and a second one in Turkey.

\section{29th ISYA, Malaysia, 2007}

The 29th International School for Young Astronomers (ISYA) was held in Malaysia (Selangor and Langkawi Island), 5-23 March 2007. ISYA 2007 was organized by the IAU with Universiti Kebangsaan Malaysia (UKM) and with the cooperation of National Space Agency of Malaysia (ANGKASA), Ministry of Science, Technology and Innovation (MOSTI) and the Universiti Malaya (UM).

The School was held at the University Kebangsaan Malaysia (UKM), Selangor, 5-9 March 2007, and subsequently at the MARA Junior Science College, Langkawi Island, 10-23 March 2007, where the National Observatory is located.

\subsection{National Organizing Committee}

The members of the Organizing Committee in Malaysia were:

- Prof. Dr. Mazlan Othman, Director General ANGKASA, Secretary General of Academy Sciences of Malaysia, Chairman of National Committee on organizing ISYA2007

- Prof. Dr. Mohd Zambri Zainuddin, Head Space Science Laboratory and Deputy Dean of Malaya University

- Prof. Dr. Baharudin Yatim, Director Space Science Institut, National University of Malaysia

- Mr. Kassim Bahali, Head Astronomy programme, Al-Khawarizmi Observatory, Malacca

- Mr. Mhd Fairos Asillam, Science Officer ANGKASA, Secretary National Organizing Committee ISYA2007.

\subsection{ISYA 2007 participants}

The number of selected participants were 38 (female 9, male 29): 10 Malaysian and 28 foreigners from 11 countries: China (2), DPR Korea (3), India (3), Indonesia (7), Nepal (1), New Zealand (1), Philippines (4), Shri Lanka (1), Taiwan (1), Thailand (3) and Vietnam (2). The students had very mixed academic backgrounds and experience ranging from a MSc degree to having finishing their $\mathrm{PhD}$. 


\subsection{ISYA academic programme}

The faculty members and lectures were:

- Dr. Chenzhou Cui, China, National Astronomical Observatory, Virtual Observatory

- Prof. Jean-Pierre De Greve, Belgium, Brussels University, Binary star evolution with massive components

- Assoc. Prof Mamoru Doi, Japan, University of Tokyo, Galaxies

- Prof. Michele Gerbaldi, France, Institut d'Astrophysique de Paris, Stellar atmosphere

- Prof. Edward Guinan, USA, Villanova University, Binary stars

- Prof. K.R. Lang, USA, Tufts University, The Sun

- Dr. Hakim L. Malasan, Indonesia, Institut Teknologi Bandung, Stellar observations

- Assoc. Prof Mark Rast, USA, University of Colorado, Astrophysics of the Sun

- Prof. N. Udaya Shankar, India, Raman Research Institute, Radioastronomy

- Prof. Mohd Zambri Zainuddin, Malaysia, Universiti Malaya, Astronomy

The ISYA students were encouraged to describe their current research. Six sessions were organized for the 27 talks given, each talk was of 15 minutes plus 5 minutes for the questions. Emphasize has been put on the Virtual Observatory and data base concepts by Dr. Chenzhou Cui. For that purpose a network of 22 computers under LINUX/Windows was set up and image processing software installed. The practical classes were based on access to real data.

The participants also had to conduct optical observations. Six observing sessions took place at the Langkawi National Observatory for imaging and spectroscopy with the robotic $0.5 \mathrm{~m}$ telescope. These observing sessions were directed by Dr. Hakim L. Malasan with M. Ridwan Hidaya, Mrs. Lau Chen Chen and M. Karzaman Ahmad. Reduction of the images and spectra taken were done under the guidance of Dr. Chenzhou Cui and Dr. Hakim L. Malasan. One half-a-day session was devoted to solar observations at the Langkawi Solar Observatory. Solar images with $\mathrm{H} \alpha$ and Ca II-K-line filters were taken under the direction of Assoc. Prof. Mark Rast and Prof. Edward Guinan.

Profs. J.-P. De Greve and Edward Guinan provided information and advice on:

- how to present results and to give a talk

- how to apply to $\mathrm{PhD}$ programme, etc.

- how to apply for jobs and writing curricula vitae / resumes, etc.

- how to write science proposals, etc.

\subsection{Closing remarks}

Besides the academic programme of the ISYA, the lecturers gave several conferences at the National Science Museum and National Planetarium, at Kuala Lumpur, at the MARA Science College, Langkawi, and at the Terengganu University.

This ISYA is the starting point for the development of new programmes in Malaysia, in particular related to the use of the Langkawi National Observatory in both stellar and solar domain. Further developments are considered in the framework of the TAD programme.

\section{30th ISYA, Turkey, 2008}

The 30th International School for Young Astronomers (ISYA 2008) was held in Istanbul, Turkey, 1-21 July 2008 It was organized with the Turkish Astronomical Society (TAD) and with the cooperation of the Yüzyil Isil School (YIS), a private primary and high school near Sariyer, Istanbul, the Istabul University (IU), and the Istanbul Kültür University. The lectures were held at the YIS (some $30 \mathrm{~km}$ from the center of Istanbul). 


\subsection{National Organizing Committee}

The members of the organizing committee in Turkey were:

- Prof. Ali Alpar, Turkish Astronomical Society and Sabanci University

- Prof. Zeki Aslan, Istanbul Kültür University

- Prof. Prof. Dursun Koer, Istanbul Kültür University

- Sinan Alis, Istanbul University, secretary

\subsection{ISYA Participants}

Out of 46 candidates, 37 were selected for participation. Five of them didn't come (2 from Egypt, 1 from Nigeria, 2 from Turkey), two additional students were added later on, bringing the actual number of participants to 35, from 12 different countries from the region. The gender distribution was $40 \%$ female, $60 \%$ male. The students had very mixed academic backgrounds and experience ranging from a MSc degree to having finished their $\mathrm{PhD}$.

\subsection{ISYA academic programme}

The faculty members and lectures were:

- Prof. Ali Alpar, Turkey, Cibanci University, Endpoints of stellar evolution, structure of neutron stars, observations of neutron stars

- Prof. Zeki Aslan, Turkey, Istanbul Kültür University, Introduction to astrometry, $C C D$ astrometry

- Prof. Jean-Pierre De Greve, Belgium, Brussels University, Structure and evolution of stars, Structure and evolution of Binaries

- Dr. Antonio Frasca, Italy, Catania Observatory, Stellar spectroscopy and data analysis with IRAF, spectroscopy of binaries

- Prof. Ed Guinan, USA, Villanova University, Binary stars as astrophysical labs, extrasolar planets and life, stellar dynamos and effects on hosted planets, research with small telescopes, and Binary stars

- Prof. Kam-Ching Leung, USA, Nebraska University, Photometry, novae, close binary observations

- Prof. Robert Williams, USA, Space Telescope Science Institute, Hubble Space Telescope science, Hubble Deep Fields, novae

- Dr. Sinan K. Yerli, Turkey, Middle East Technical University, CCD reduction techniques using IRAF with hands-on computer training on real/archive data

In the second week the students had to give the topics for their presentations and a schedule was developed within the slots foreseen in the third week. Most of the students consulted Ed Guinan and/or Jean-Pierre De Greve for the content of their powerpoint presentation. The presentations took place on 5 slots of 1.5 hour from Tuesday till Saturday. For each student 15 minutes were foreseen for the presentation and the discussion. The lecturers agreed that the presentations were well prepared and of high quality.

Attention in the program was devoted to the development of observing competencies. Thus, an important aspect of the first week was the preparation of the observations to be carried out in the second week. With the support of Dr. Paul Roche, ISYA had access to the $2 \mathrm{~m}$ robotic Faulkes telescope on Maoui, Hawaii. Three slots of 2 hours were offered from $3.30 \mathrm{pm}$ to $5.30 \mathrm{pm}$ (Istanbul time) on Monday, Wednesday and Friday of the second week (through the appreciated support of Paul Roche). In the first week the students were asked to organize themselves into teams and to develop and propose a feasible observing project. In the second week slots of ten minutes were given to each team within the two-hour slots. In the lectures the students gained insight in the IRAF 
software, to be able to carry out proper reductions of the data. In the third week, students could reduce their data.

Profs. J.-P. De Greve and Edward Guinan provided information and advice on:

- how to present results and to give a talk

- how to apply to $\mathrm{PhD}$ programme, etc.

- how to apply for jobs and writing curricula vitae / resumes, etc.

- how to write a scientific paper and getting it published.

Besides the academic programme of the ISYA, Jean-Pierre De Greve assisted to a Saturday morning television programme on one of the Turkish television channels.

The cultural programme for the students consisted of a two trips to the center of Istanbul, to visit the Aya Sofia, the Imperial Palace, a visit to the Sultan's Palace, the Cistern and the Blue Mosque, and a sightseeing boat trip through the Bosphorus. A third visit and fourth visit offered a day at the grand bazaar and a visit to Istanbul's modern shopping area.

\subsection{Closing remarks}

During the ISYA in Turkey the young participants discussed future plans among each other and with the lecturers, and developed an intercultural network of young astronomers to help or advise each other in the future.

\section{News Release - IAU0803: The International Astronomical Union teams up with the Norwegian Academy of Science and the Kavli Prize}

June 5, 2008, Oslo

In an important move, the Norwegian Academy of Science and Letters and the International Astronomical Union have joined forces in support of both the Kavli Prize and the IAU International Schools for Young Astronomers.

The International Astronomical Union (IAU) and the Norwegian Academy of Sciences and Letters (NASL) have united in support of the Kavli Prize and the IAU International Schools for Young Astronomers (ISYA) after finding common ground in promoting astrophysics research and training young scientists in the latest astronomical research techniques.

The Kavli Prize Committee is composed of distinguished scientist based on recommendations from leading international academies and other equivalent scientific organizations. Beginning in 2010, the Norwegian Academy will seek advice by the International Astronomical Union in order to establish a balanced prize committee with respect to the various fields of Astrophysics. They will work together on publicising the events. From 2009 NASL will donate a yearly sum to the IAU ISYA programme, allowing the programme to expand. Furthermore, Kavli Prize winners will be invited to speak at the ISYA alongside the usual experts. The agreement was signed by the President of NASL, Prof. Ole Didrik Laerum, and the President of the IAU, Dr. Catherine J. Cesarsky.

The Kavli Prize, which is given in the areas of astrophysics, neuroscience and nanoscience, encourages future research directly through incentive by providing winners with $\$ 1,000,000$, a medal and a diploma. Every two years the Kavli Prize in Astrophysics is awarded to one or more scientists who have demonstrated outstanding achievement in 
advancing human knowledge and understanding of the origin, evolution, and properties of the vast Universe. The Prize is open to a wide range of fields, including cosmology, astrophysics, astronomy, planetary science, solar physics, space science, astrobiology, astronomical and astrophysical instrumentation and particle astrophysics.

The first Kavli Prize in Astrophysics was awarded jointly to Maarten Schmidt, of the California Institute of Technology in the United States, and to Donald Lynden-Bell, of Cambridge University in the United Kingdom, on 28 May 2008.

Since 1967, the IAU ISYA programme has promoted the development of professional astronomers around the world by facilitating interaction between young scholars and established professionals. It also provides them with the practical knowledge essential to access the data archives that have become the basis for astronomical research. Individuals with a Masters degree in science can participate in the 21-day conference, which occurs at a different location each year to perpetuate international cooperation in astronomical research. Participants do everything from listen to lectures given by experts to becoming involved through hands-on practicals and computer sessions where they learn how to use essential astronomical tools.

This year the ISYA meeting will take place in Istanbul, Turkey in July. A diverse array of topics will be covered, from the structure of neutron stars to a discussion of the science completed using the Hubble Space Telescope.

- End of Press Release -

Jean-Pierre De Greve \& Michèle Gerbaldi chair and past-chair of the Program Group

\section{References}

Gerbaldi, M. 2008, in: K. A. van der Hucht (ed.), Transactions IAU XXVIB (Cambridge: CUP), p. 238 